\title{
Searches for additional neutral Higgs bosons at CMS
}

\author{
Dermot Moran*† \\ CIEMAT, Av. Complutense, 40, 28040 Madrid, Spain \\ E-mail: dermot.anthony.moran@cern.ch
}

\begin{abstract}
Searches for additional neutral Higgs bosons by the CMS experiment at the LHC are presented. A number of different decay channels and production mechanisms are covered. The analyses are based on proton-proton collisions at $\sqrt{s}=13 \mathrm{TeV}$ recorded in 2016, corresponding to an integrated luminosity of $35.9 \mathrm{fb}^{-1}$. The observed data are consistent with the standard model expectations. Upper limits on the Higgs boson production cross section times branching ratio are set for each search, typically in the context of a two-Higgs-doublet model formulation. These results further reduce the allowed parameter space for extensions of the standard model.
\end{abstract}

European Physical Society Conference on High Energy Physics - EPS-HEP2019 -

10-17 July, 2019

Ghent, Belgium

\footnotetext{
* Speaker.

${ }^{\dagger}$ On behalf of the CMS Collaboration.
} 


\section{Introduction}

The discovery of the standard model (SM) Higgs boson with a mass close to $125 \mathrm{GeV}[1,2,3]$, denoted here as $\mathrm{h}(125)$, has led to a new era in particle physics. In order to determine whether the SM gives a complete description of the Higgs sector, precise measurements of the $h(125)$ coupling strengths, CP structure and kinematic distributions are required. A complementary and important strategy involves the search for additional Higgs bosons whose existence would prove the presence of beyond-the-SM (BSM) physics in the form of a non minimal Higgs sector. The search for additional scalar resonances remains one of the main objectives at the LHC.

One of the simplest extensions of the SM Higgs sector is known as the two-Higgs-doubletmodel (2HDM) [4] and involves the introduction of a second scalar doublet. Various formulations of the 2HDM predict different couplings of the two doublets to right-handed quarks and charged leptons. There are five physical Higgs bosons predicted: two $\mathrm{CP}$-even neutral bosons $\mathrm{h}$ and $\mathrm{H}$; a neutral CP-odd boson $\mathrm{A}$; and two charged bosons $\mathrm{H}^{ \pm}$. In most formulations of the $2 \mathrm{HDM}, \mathrm{h}$ corresponds to the $\mathrm{h}(125)$, and $\mathrm{H}$ is an additional heavy CP-even Higgs boson. The 2HDM has two important free parameters, $\alpha$ and $\tan \beta$, which are the mixing angle and the ratio of the vacuum expectation values of the two Higgs doublets, respectively. In the alignment limit, which occurs at $\cos (\beta-\alpha)=0$, the properties of $\mathrm{h}$ approach those of the SM Higgs boson.

A popular BSM theory is the minimal supersymmetric standard model (MSSM) [5] which incorporates a Type-II 2HDM. In the Type-II formulation [4] the up-type quarks couple to a different doublet than the down-type quarks and leptons. At tree level, the whole phenomenology can be described using just two parameters. By convention, these parameters are chosen to be $\tan \beta$ and $\mathrm{m}_{\mathrm{A}}$, the mass of the pseudoscalar Higgs boson. Beyond the tree level, the MSSM Higgs sector depends on additional parameters which enter via higher-order corrections in perturbation theory, and which are usually fixed to values motivated by experimental constraints and theoretical assumptions. Benchmark scenarios are defined by setting these parameters such that a wide range of the parameter space is compatible with the $\mathrm{h}(125)$ mass and production rate measurements, and with limits from searches for supersymmetry particles and additional Higgs bosons at the LHC.

In this report, searches for additional neutral Higgs bosons by the CMS experiment at the LHC [6], covering a number of different decay channels and production mechanisms, are presented. The analyses are based on proton-proton collisions at $\sqrt{s}=13 \mathrm{TeV}$ recorded in 2016, corresponding to an integrated luminosity of $35.9 \mathrm{fb}^{-1}$.

\section{Search for $\mathrm{H} \rightarrow \mathrm{ZA} \rightarrow \ell^{+} \ell^{-} \mathrm{b} \overline{\mathrm{b}}$}

In the 2HDM alignment limit, and assuming twisted custodial symmetry, one of the largest branching fractions for a heavy scalar $\mathrm{H}$ is for the decay to a lighter pseudoscalar $\mathrm{A}$ and a $\mathrm{Z}$ boson. In this context a search is performed for $\mathrm{H} \rightarrow \mathrm{ZA}$ where the $\mathrm{Z}$ decays into an opposite-sign electron or muon pair, and the light Higgs boson into a b quark pair [7].

The invariant mass distributions of the $\ell \ell b \bar{b}$ and $b \bar{b}$ systems, $m_{\ell \ell j \mathrm{j}}$ and $\mathrm{m}_{\mathrm{jj}}$, are used to search for a resonant-like excess compatible with the $\mathrm{H}$ and $\mathrm{A}$ bosons. As these distributions are inherently positively correlated, an elliptical signal region in the $\mathrm{m}_{\ell \ell \mathrm{jj}}-\mathrm{m}_{\mathrm{jj}}$ plane is chosen. The ellipses have different sizes and tilt angles depending on the signal hypothesis under consideration. Selected 
events in the $\mathrm{m}_{\ell \ell \mathrm{jj}}-\mathrm{m}_{\mathrm{jj}}$ plane are classified in six regions around the center of the ellipse defined for each signal hypothesis. The size of the elliptical regions is set using a dilation parameter $\rho$, where $1 \rho$ corresponds to roughly $1 \sigma$ of the signal resolution. The regions are built in $\rho$ steps of 0.5 , from 0 to 3 , and lead to a template containing six bins used to perform the statistical analysis.

Two categories are defined based on the flavour of the lepton pairs. The dominant Z+Jets background process is estimated from next-to-leading order (NLO) simulation, while a different-flavour lepton pair control region is used to estimate the top quark background. No significant deviations from the SM expectations are observed. The left hand plot of Figure 1 shows the exclusion limits in the $\mathrm{m}_{\mathrm{A}}-\mathrm{m}_{\mathrm{H}}$ plane for a $2 \mathrm{HDM}$ benchmark with $\tan \beta=1.5$ and $\cos (\beta-\alpha)=0.01$. Limits are also set for the case of a non-twisted custodial symmetry scenario, characteristic of the MSSM, where the masses of the $\mathrm{A}$ and $\mathrm{H}$ bosons are exchanged. The right hand plot of Figure 1 shows the exclusion limits in the $\cos (\beta-\alpha)-\tan \beta$ plane for $\mathrm{m}_{\mathrm{H}}=379 \mathrm{GeV}$ and $\mathrm{m}_{\mathrm{A}}=172 \mathrm{GeV}$.
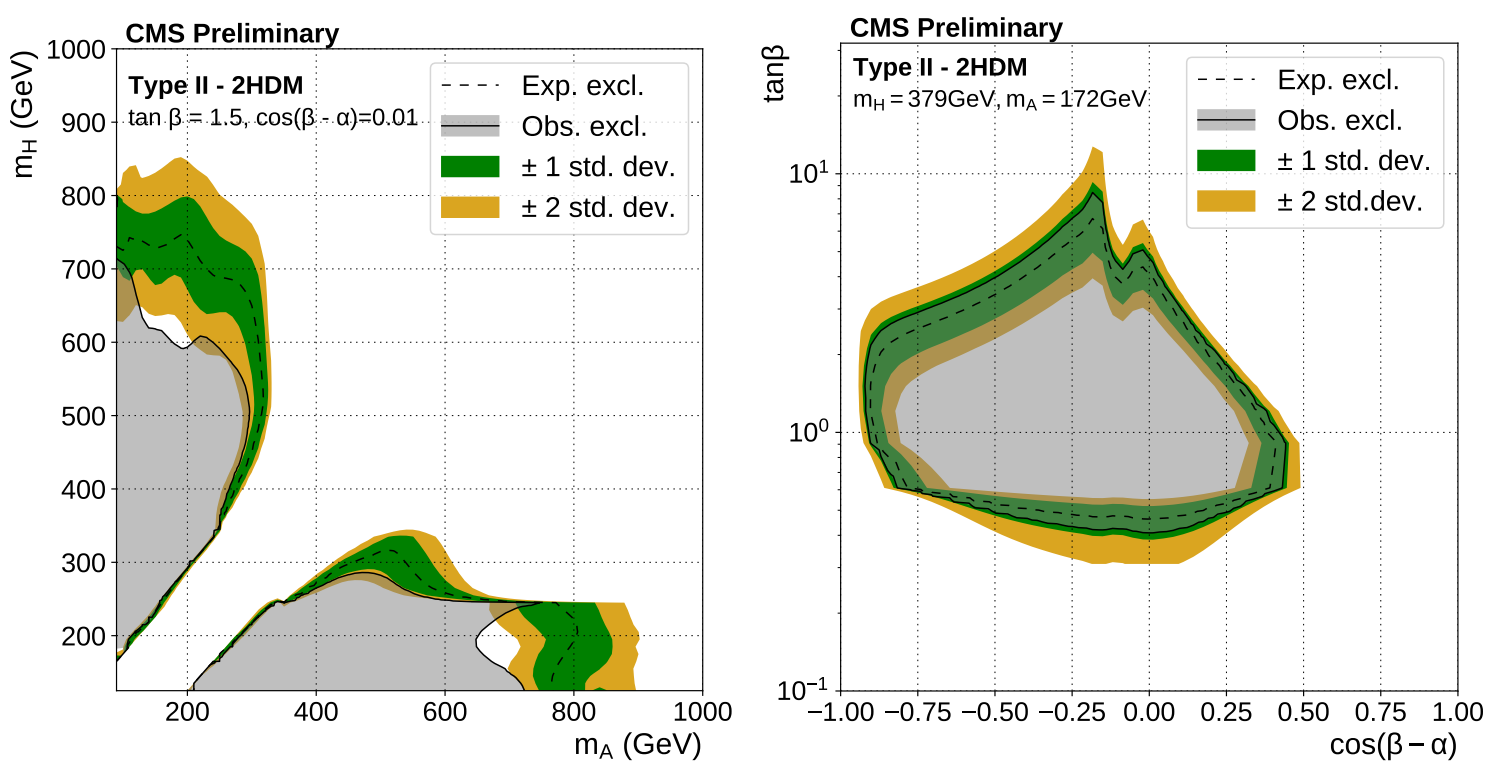

Figure 1: Exclusion limits in the $\mathrm{m}_{\mathrm{A}}-\mathrm{m}_{\mathrm{H}}$ plane for a $2 \mathrm{HDM}$ benchmark with $\tan \beta=1.5$ and $\cos (\beta-\alpha)=$ 0.01 (left), and in the $\cos (\beta-\alpha)-\tan \beta$ plane for $\mathrm{m}_{\mathrm{H}}=379 \mathrm{GeV}$ and $\mathrm{m}_{\mathrm{A}}=172 \mathrm{GeV}$ (right) [7].

\section{Search for lepton flavour violating $H \rightarrow \mu \tau$ and $H \rightarrow e \tau$}

A search has been performed for lepton flavor violating (LFV) decays of a heavy Higgs boson in the $\mu \tau_{h}, \mu \tau_{e}, e \tau_{h}$ and $e \tau_{\mu}$ channels [8], where $\tau_{h}, \tau_{e}$ and $\tau_{\mu}$ correspond to the hadronic, electronic and muonic decay channels of $\tau$ leptons, respectively. The collinear mass of the Higgs boson candidate is determined as $\mathrm{M}_{\text {coll }}=\mathrm{M}_{\mathrm{vis}} / \sqrt{\mathrm{x}_{\tau}^{\text {vis }}}$, where $\mathrm{M}_{\mathrm{vis}}$ is the invariant mass of the visible decay products of the $\mu \tau$ or $e \tau$ system and $\mathrm{x}_{\tau}^{\mathrm{vis}}$ is the fraction of energy carried by the visible decay products of the $\tau$. The $\mathrm{x}_{\tau}^{\mathrm{vis}}$ is estimated assuming that, since $\mathrm{m}_{\mathrm{H}}>>\mathrm{m}_{\tau}$, the $\tau$ lepton decay products are highly Lorentz boosted in the direction of the $\tau$ candidate, therefore the neutrino momenta can be approximated to have the same direction as the other visible decay product.

The final selection criteria are optimized separately for each decay mode in the $\mathrm{m}_{\mathrm{H}}$ ranges 200 $<\mathrm{m}_{\mathrm{H}}<450 \mathrm{GeV}$ and $450<\mathrm{m}_{\mathrm{H}}<900 \mathrm{GeV}$. Events are then divided into 0 -jet and 1-jet categories 
according to the number of jets in the event. The most significant backgrounds come from $\mathrm{W}+\mathrm{jets}$ and QCD multijet production, where one or two jets are misidentified as leptons. The misidentified lepton probability is estimated from $\mathrm{Z}+$ jets events in data.

A maximum likelihood fit is performed to the $\mathbf{M}_{\text {coll }}$ distributions and no significant excess over the background-only hypothesis is observed in any category. Figure 2 shows the resulting upper limits on the heavy Higgs boson production cross section times branching ratio to LFV lepton pairs.
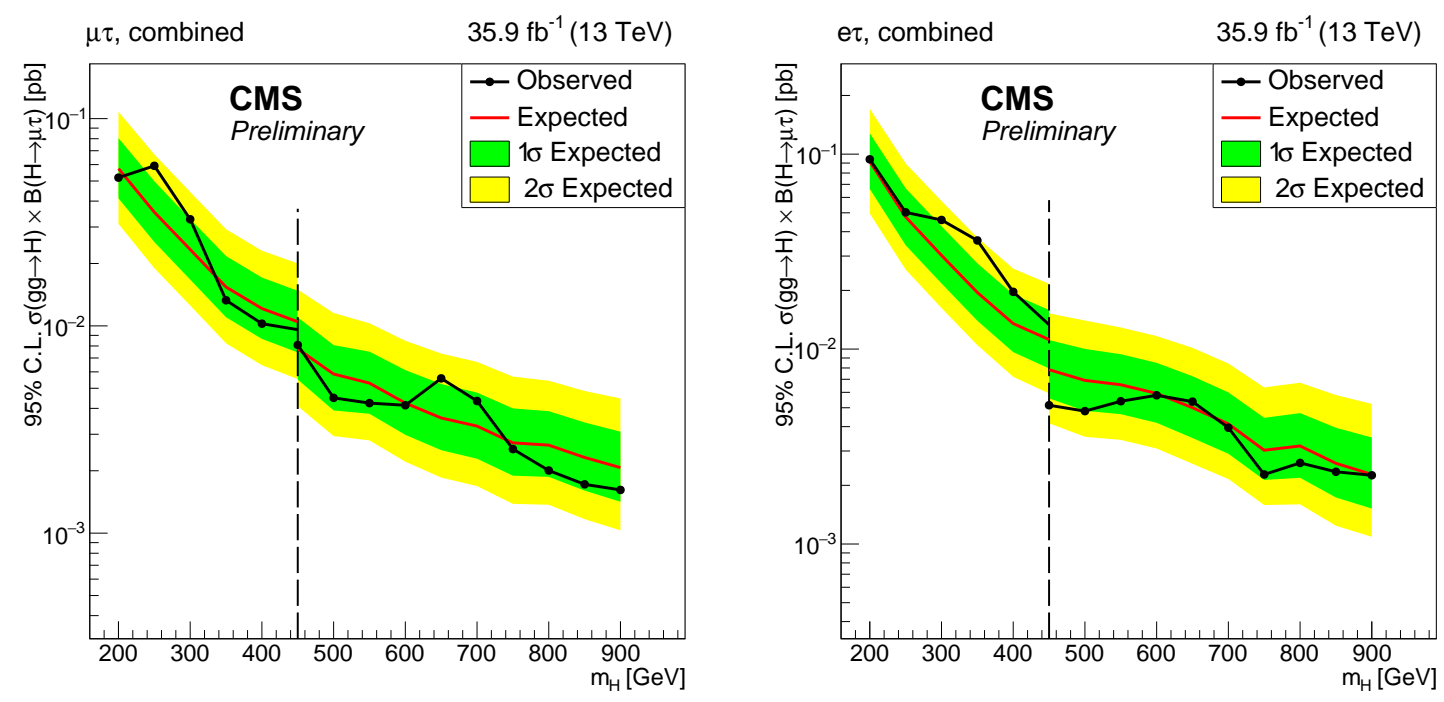

Figure 2: Upper limits the heavy Higgs boson production cross section times branching ratio to LFV lepton pairs $\mu \tau$ (left) and $e \tau$ (right) [8].

\section{Search for $b \bar{b}$ associated production of low mass $A \rightarrow \tau \tau$}

In this analysis a search for a low-mass pseudoscalar Higgs boson A, produced in association

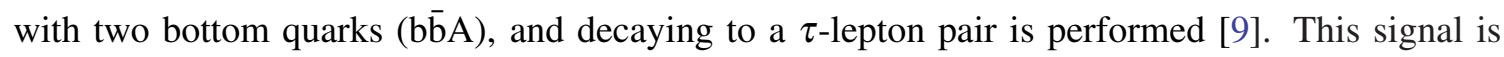
enhanced when the Yukawa couplings of A to down-type fermions are negative (wrong-sign). The search is conducted in the semileptonic $\tau$-lepton pair $\tau_{\mu} \tau_{h}$ and $\tau_{e} \tau_{h}$ final states. A mass range between 20 and $70 \mathrm{GeV}$ is considered, bounded below by kinematic requirements and above by the background of the $\mathrm{Z}$ mass peak. Selected events are required to have a central b-tagged jet.

The variable $\mathrm{D}_{\zeta}$ [10] quantifies the compatibility of events with the topology wherein the direction of neutrinos from the $\tau$-lepton decays are aligned with the direction of the visible $\tau$ lepton decay products. This variable is used to suppress the $\mathrm{W}+\mathrm{jets}$ and top quark backgrounds, both of which are estimated from simulation with the predictions verified in data control regions. The QCD multijet background is small and is estimated using a same-sign $\tau$-lepton pair control region.

An excess of events over the SM backgrounds is searched for using the invariant mass of the $\tau$-lepton pair, constructed using the $\mathrm{SV}_{\text {fit }}$ mass algorithm [11]. The data are consistent with the background-only hypothesis of the SM. Figure 3 shows the upper limits on the pseudoscalar Higgs boson production cross section times branching ratio to $\tau$-lepton pairs as a function of $\mathrm{m}_{\mathrm{A}}$. A 
number of representative $2 \mathrm{HDMs}$ with variations of the $\tan \beta$ and $\mathrm{m}_{\mathrm{A}}$ parameters are also shown for SM-like and wrong-sign Yukawa couplings to the down-type fermions. Most of the wrong-sign models are excluded by the current data.

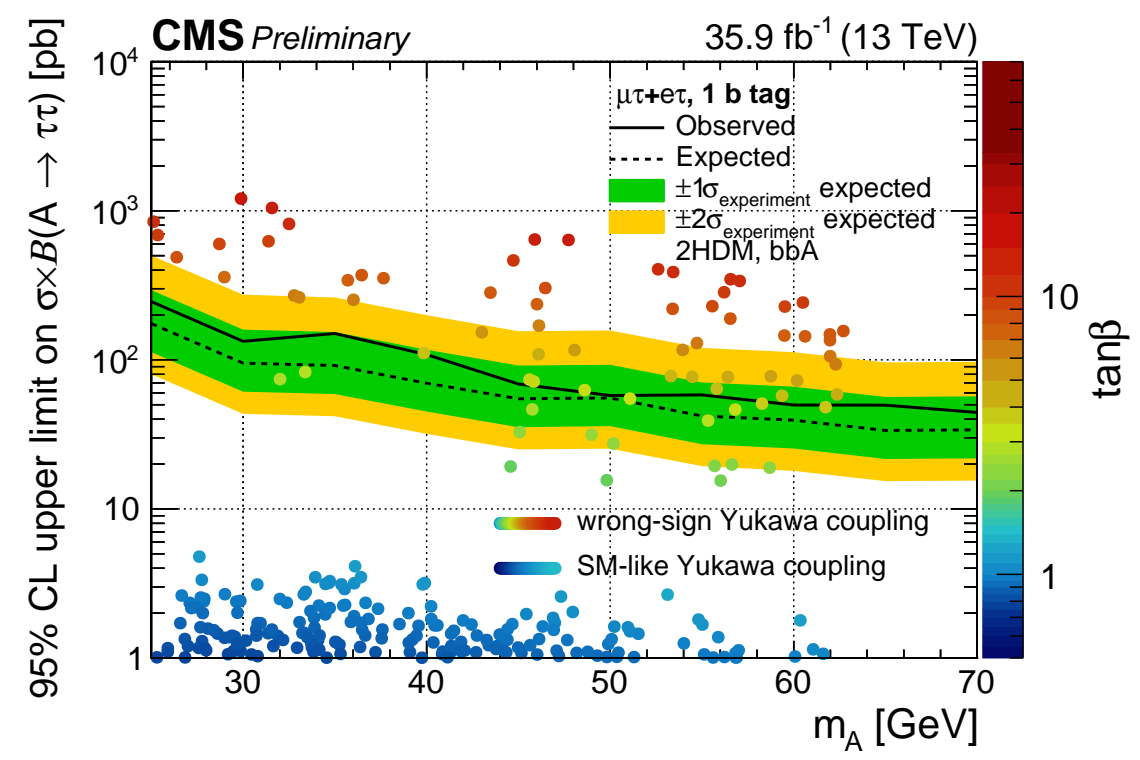

Figure 3: Upper limits on the pseudoscalar Higgs boson production cross section times branching ratio to $\tau$-lepton pairs as a function of $\mathrm{m}_{\mathrm{A}}[9]$.

\section{Search for $\mathrm{H} \rightarrow \mathrm{WW}$}

A search for a heavy Higgs boson decaying to a pair of W bosons in the mass range from 200 $\mathrm{GeV}$ to $3 \mathrm{TeV}$ is presented [12]. The decay of the $\mathrm{W}$ boson pair is reconstructed in the $2 \ell 2 v$ and $\ell v 2 q$ final states. Both gluon fusion and vector boson fusion production of the signal are considered, with a number of hypotheses for their relative contribution considered. Interference effects between the signal and background are also taken into account. Dedicated event categorizations based on the kinematic properties of associated jets and matrix element techniques are employed to optimise the signal sensitivity.

For the semi-leptonic channel, the hadronically decaying $\mathrm{W}$ boson may be reconstructed as two quark-jets (a dijet) or as a single high $\mathrm{p}_{\mathrm{T}}$ merged jet with substructure techniques used to discriminate vector bosons from QCD jets. A $\mathrm{W}$ mass constraint is applied to the $\ell v$ system to estimate the $\mathrm{p}_{\mathrm{z}}$ component of the missing momentum. The hadronic and leptonic $\mathrm{W}$ decay candidates are then combined into WW resonance candidates, the final discriminating variable is the invariant mass of the WW system. The dominant backgrounds are from $\mathrm{W}+\mathrm{Jets}$ and top quark production.

Both the same flavour and different flavour final states are considered in the fully leptonic channel. The final discriminating variable used is the visible Higgs mass reconstructed with the dilepton four-momentum and the measured missing momentum in the event. The dominant backgrounds in this channel are from non-resonant WW and top quark production, with Drell-Yan production also a significant background in the same-flavour category. 
The dominant backgrounds are modelled by simulation with their normalisations determined using control regions in data. No evidence for an excess of events with respect to the SM predictions is observed. The left hand plot of Figure 4 shows the upper limits on the heavy Higgs boson cross section times branching fraction to two $\mathrm{W}$ bosons, the fraction of the VBF production cross section with respect to the total cross section has been allowed to float in the fit to the data. The right hand plot of Figure 4 shows the exclusion limits for a heavy Higgs bosons in the $\tan \beta-\mathrm{m}_{\mathrm{A}}$ plane for the hMSSM benchmark scenario [13].
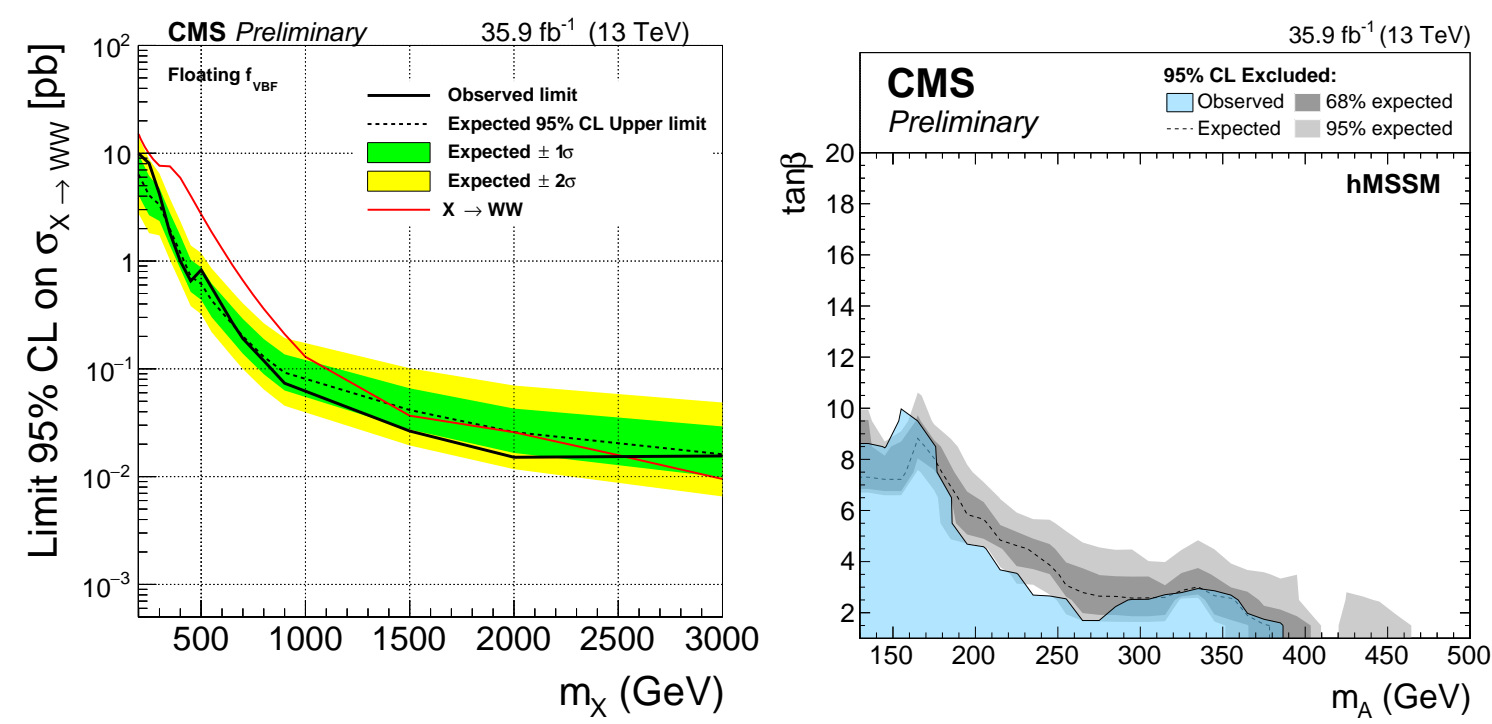

Figure 4: Upper limits on the heavy Higgs boson cross section times branching fraction to two W bosons (left) and exclusion limits for a heavy Higgs bosons in the $\tan \beta-\mathrm{m}_{\mathrm{A}}$ plane for the hMSSM benchmark scenario (right) [12].

\section{Summary}

A number of searches for additional neutral Higgs bosons have been carried out by the CMS experiment using 2016 data at a center-of-mass energy of $13 \mathrm{TeV}$. No evidence for beyond-theSM physics has been observed. Upper limits on the Higgs boson production cross section times branching ratio are set for each search, typically in the context of a two-Higgs-doublet model formulation. These results further reduce the allowed parameter space for extensions of the standard model. All the searches presented are being repeated with the full Run 2 dataset which corresponds to an integrated luminosity of roughly $137 \mathrm{fb}^{-1}$. The increase in integrated luminosity will give a substantial boost to the sensitivity of searches for new physics phenomena in the Higgs sector. 


\section{References}

[1] ATLAS Collaboration, Observation of a new particle in the search for the standard model Higgs boson with the ATLAS detector at the LHC, Phys. Lett. B 716 (2012) 1.

[2] CMS Collaboration, Observation of a new boson at a mass of $125 \mathrm{GeV}$ with the CMS experiment at the LHC, Phys. Lett. B 716 (2012) 30.

[3] CMS Collaboration, Observation of a new boson with mass near $125 \mathrm{GeV}$ in pp Collisions at $\sqrt{\mathrm{s}}=7$ and $8 \mathrm{TeV}, \mathrm{JHEP} 06$ (2013) 081.

[4] Branco, G. C. and Ferreira, P. M. and Lavoura, L. and Rebelo, M. N. and Sher, M. and Silva, J. P., Theory and phenomenology of two-Higgs-doublet models, Phys. Rept. 516 (2012) 1.

[5] Fayet, Pierre, Supergauge invariant extension of the Higgs mechanism and a model for the electron and its neutrino, Nucl. Phys. B 90 (1975) 104.

[6] CMS Collaboration, The CMS experiment at the CERN LHC JINST 3 (2008) S08004.

[7] CMS Collaboration, Search for 2 HDM neutral Higgs bosons through the $\mathrm{H} \rightarrow \mathrm{ZA} \rightarrow \ell^{+} \ell^{-} \mathrm{b} \overline{\mathrm{b}}$ process in proton-proton collisions at $\sqrt{s}=13 \mathrm{TeV}$, CMS-PAS-HIG-18-012 (2019).

[8] CMS Collaboration, Search for lepton flavour violating decays of neutral heavy Higgs boson to $\mu \tau$ and e $\tau$ in proton-proton collisions at $\sqrt{s}=13 \mathrm{TeV}, C M S-P A S-H I G-18-017$ (2019), https://cds.cern.ch/record/2674882.

[9] CMS Collaboration, Search for a low-mass $\tau^{-} \tau^{+}$resonance in association with a bottom quark in proton-proton collisions at $\sqrt{s}=13 \mathrm{TeV}$, JHEP 05 (2019) 210.

[10] CDF Collaboration, Search for neutral MSSM Higgs bosons decaying to tau pairs in p p collisions at $\sqrt{s}=1.96$ TeV, Phys. Rev. Lett. 96 (2006) 011802.

[11] Bianchini, Lorenzo and Conway, John and Friis, Evan Klose and Veelken, Christian, Reconstruction of the Higgs mass in $H \rightarrow \tau \tau$ Events by Dynamical Likelihood techniques, J. Phys. Conf. Ser. 513 (2014) 022035.

[12] CMS Collaboration, Search for a heavy Higgs boson decaying to a pair of W bosons in proton-proton collisions at $\sqrt{s}=13 \mathrm{TeV}$, CMS-PAS-HIG-17-033 (2019), https://cds.cern.ch/record/2668687.

[13] Djouadi, A. and Maiani, L. and Moreau, G. and Polosa, A. and Quevillon, J. and Riquer, V., The post-Higgs MSSM scenario: Habemus MSSM?, Eur. Phys. J. C73 (2013) 2650. 